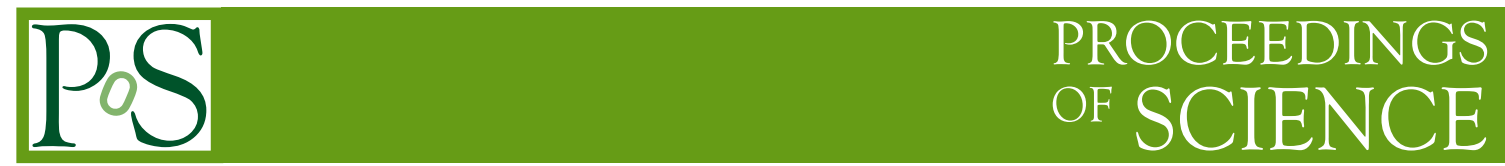

\title{
Momentum and position variables in light-front Hamiltonians and wave functions
}

\section{S. D. Głazek}

University of Warsaw, Poland

E-mail: stglazekefuw.edu.pl

\begin{abstract}
Momentum variables $p=x P+k$ are not simply related through a Fourier transform to the spacetime position of particles on the LF because momentum component + is only positive and $x$ is limited to the range between 0 and 1 . Nevertheless, $x$ and $k$ can be handled in a way which leads to visualization of non-local renormalized LF Hamiltonian interaction densities and wave functions in position representation. The same way of handling $x$ and $k$ also shows how local interactions emerge from non-local renormalized Hamiltonians when the renormalization group scale parameter of an effective theory tends to infinity.
\end{abstract}

Light Cone 2010: Relativistic Hadronic and Particle Physics

June 14-18, 2010

Valencia, Spain 


\section{Introduction}

Light-front quantization formalism is typically applied to quantum field theory (QFT) using momentum variables, i.e., using a Fock space whose basis is built by applying creation operators of particles with definite momentum components $p^{+}$and $p^{\perp}$ (in conventional notation, where $x^{ \pm}=$ $x^{0} \pm x^{3}$ and LF hyperplane in space-time is defined by condition $\left.x^{+}=0\right)$ to a state of vacuum. For example, a constituent momentum $p$ in a proton of momentum $P$ is typically parameterized by writing $p=x_{B j} P+k$, as in the infinite momentum frame $[1,2,3,4,5,6]$, where $x_{B j}$ denotes $^{1}$ the fraction $p^{+} / P^{+}$and $k$ has only $\perp$ components different from 0 . Position representation using quantum fields as functions of $x^{-}$and $x^{\perp}$ on the LF is rarely used. The trouble with going to position variables can be seen by looking at two equations,

$$
\begin{aligned}
& \hat{\psi}\left(x^{\perp}\right)=\int d^{2} k^{\perp} e^{i k^{\perp} x^{\perp}} \psi\left(k^{\perp}\right), \\
& \hat{\psi}\left(x^{-}\right)=\int_{0}^{1} P^{+} d x_{B j} e^{i x_{B j} P^{+} x^{-} / 2} \psi\left(x_{B j}\right),
\end{aligned}
$$

which relate probability amplitudes (wave functions) in momentum and position variables. While $\perp$ momentum is integrated from $-\infty$ to $+\infty$, the + momentum is integrated only from 0 to $P^{+}$, the total available $P^{+}$being conserved by the dynamics. Eq. (1.1) is similar to the Fourier relationship known in the instant form (IF) of dynamics, while Eq. (1.2) is odd and seems to exclude thinking in terms of localized positions of particles on the LF in direction of $x^{-}$, despite that canonical field-theoretic analysis is possible [7,8].

The issue of localization of particles in $x^{-}$arises when one attempts to construct renormalized quantum field theory on the LF using power-counting to identify all possible interaction terms [9]. For example, when one attempts to introduce a confining potential, the question arises how this potential should depend on the LF position variables. There is also an intriguing recent discovery [10] that wave functions of hadrons in LF QCD may be encoded using ideas based on AdS/CFT duality. Strictly speaking, the duality attempts to identify a local (conformal) QFT in a 4-dimensional position space as a boundary region of a richer theory in a 5-dimensional AdS space. It has been suggested $[11,12,13]$ that in theories such as QCD, in which scale invariance is broken, the 5th dimension should be understood as corresponding to a renormalization group (RG) scale parameter. In this context, the question arises [14] if and how the hypothesis of connection between the 5th dimension and RG parameter can be verified using the RG approach of the type outlined in Ref. [9] in LF QCD. However, in order to have a chance of discussing a renormalized LF QFT in 4 dimensions a la [9] and extend the discussion to 5 dimensions using appropriate RG procedure, one needs a clear concept of how the renormalized picture of quanta in momentum representation translates to position space. This is needed to investigate structure of corresponding Hamiltonian densities in position space and finding out if they can correspond to Lagrangian densities that are used in duality arguments. The connection between momentum and position representations in LF dynamics is the subject of this note. Explanation of elements used in the following discussion can be found in [15].

We first discuss the structure of renormalized Hamiltonian interaction densities on the LF. They are non-local and the width of their non-locality on the LF hyperplane in space-time is pa-

\footnotetext{
${ }^{1}$ Symbol $x$ has subscript ${ }_{B j}$ to distinguish the momentum fraction from $x$ reserved here for position variables.
} 
rameterized by $1 / \lambda$, where $\lambda$ is the RG scale parameter with dimension of momentum. Then we explain on a generic example of a three fields vertex how locality emerges when $\lambda \rightarrow \infty$ despite that momenta $p^{+}$of particles in LF dynamics are limited to a finite interval instead of extending from $-\infty$ to $+\infty$, as it happens in the case of $p^{z}$ in the IF of dynamics. The Hamiltonian densities are eventually related to wave functions and we conclude with general comments regarding the resulting structures.

\section{How locality emerges in renormalized LF Hamiltonians}

How locality emerges in renormalized LF Hamiltonians is addressed here using renormalization group procedure for effective particles (RGPEP) [15]. The starting point is a local canonical theory. RGPEP generates a family of Hamiltonians parameterized by $\lambda$, and it is designed so that $\lambda$ is a momentum width of form factors in interaction vertices. Canonical theory with counterterms corresponds to $\lambda=\infty$. Physically important examples of canonical Hamiltonian interaction terms are given by integrals of their densities over the LF hyperplane, $H_{I}=\int d^{3} x \mathscr{H}_{I}(x)$. For example,

$$
\begin{aligned}
\mathscr{H}(x)_{\text {fermion-gaugeboson }} & =g: \bar{\psi}(x) \not A(x) \psi(x): \\
\mathscr{H}(x)_{\text {fermion-scalarboson }} & =g: \bar{\psi}(x) \phi(x) \psi(x):, \\
\mathscr{H}(x)_{\text {non-abelian gaugebosons }} & =g: \operatorname{Tr} \partial_{\mu} A_{v}(x)\left[A^{\mu}(x), A^{v}(x)\right]:,
\end{aligned}
$$

and all fields have the same space-time argument. ${ }^{2}$ From these local densities, application of RGPEP renders at scale $\lambda$ non-local interactions of the form

$$
H_{\lambda I}=\int d^{3} x_{1} d^{3} x_{2} d^{3} x_{3} \mathscr{H}_{\lambda I}\left(x_{1}, x_{2}, x_{3}\right)
$$

where the integrations extend over the LF. Instead of dealing with different kinds of fields separately, we introduce here a generic field $\psi(x)$ and assume for simplicity of discussion that it is a Hermitian scalar field. In this notation, a non-local three fields interaction term at scale $\lambda$ reads

$$
\mathscr{H}_{\lambda I}\left(x_{1}, x_{2}, x_{3}\right)=g_{\lambda} \hat{f}_{\lambda}\left(x_{1}, x_{2}, x_{3}\right): \psi_{\lambda}\left(x_{1}\right) \psi_{\lambda}\left(x_{2}\right) \psi_{\lambda}\left(x_{3}\right):
$$

where $g_{\lambda}$ is an effective coupling constant, $\hat{f}_{\lambda}\left(x_{1}, x_{2}, x_{3}\right)$ is the RGPEP form factor in position space, and $\psi_{\lambda}(x)$ is a quantum field operator for effective particles (see below).

RGPEP introduces the form factor $\hat{f}_{\lambda}$ in a way that can be illustrated in first-order perturbation theory in a specific version using beautiful Wegner's equation [16]. One uses parameter $s=1 / \lambda^{2}$ and writes the equation for $H_{s}=H_{0}+H_{I s}$ as (the subscript $s$ is dropped)

$$
\frac{d}{d s} H=\left[\left[H_{0}, H\right], H\right]
$$

while the initial condition for $s=0$, or $\lambda=\infty$, is given by the matrix of canonical Hamiltonian with all due counterterms, $H(0)=H_{c a n}+C T$. Keeping only linear terms in the interaction on the right-hand side, $\left[\left[H_{0}, H\right], H\right]=\left[\left[H_{0}, H_{I}\right], H_{0}\right]+O\left(H_{I}^{2}\right) \sim-H_{0}^{2} H_{I}+2 H_{0} H_{I} H_{0}-H_{I} H_{0}^{2}$, one obtains

\footnotetext{
${ }^{2}$ The densities contain constrained field components that are not determined locally even in free field theory, but the expressions one obtains in terms of the complete free fields in the listed examples, are local.
} 
an elementary set of equations for matrix elements of $H$ in the basis built from eigenstates of $H_{0}$, $\frac{d}{d s} H_{\text {Imn }} \sim-\left(E_{m}-E_{n}\right)^{2} H_{\text {Imn }}$, with a simple solution

$$
H_{\text {Imn }}(s) \sim e^{-s\left(E_{m}-E_{n}\right)^{2}} H_{\operatorname{Imn}}(0) .
$$

In the IF of dynamics, this result amounts to multiplication of interaction vertices by the form factor $f_{\lambda}=e^{-(\Delta E / \lambda)^{2}}$, where $\Delta E=E_{m}-E_{n}$ denotes the change of free energy across the interaction term. In full RGPEP in LF dynamics, this result translates [15] to a form factor $f_{\lambda}=e^{-\left(\Delta \mathscr{M}^{2} / \lambda^{2}\right)^{2}}$, in which $\Delta \mathscr{M}^{2}$ denotes the change of invariant mass squared of interacting particles across the interaction vertex.

The LF RGPEP equations are actually set up using a unitary rotation of creation and annihilation operators for canonical, point-like particles into similar operators for effective particles of size $1 / \lambda$. Thus, if a canonical interaction term has the structure corresponding to $g \psi^{3}$ theory

$$
H_{I}=3 g \int\left[p_{1} p_{2} p_{3}\right]\left(a_{p_{1}}^{\dagger} a_{p_{2}}^{\dagger} a_{p_{3}}+\text { h.c. }\right) 2(2 \pi)^{3} \delta^{3}\left(p_{1}+p_{2}-p_{3}\right),
$$

the renormalized non-local term of first order in the effective coupling constant has the structure

$$
H_{\lambda I}=3 g_{\lambda} \int\left[p_{1} p_{2} p_{3}\right] f_{\lambda}\left(a_{\lambda p_{1}}^{\dagger} a_{\lambda p_{2}}^{\dagger} a_{\lambda p_{3}}+\text { h.c. }\right) 2(2 \pi)^{3} \delta^{3}\left(p_{1}+p_{2}-p_{3}\right),
$$

where $f_{\lambda}=e^{-\left[\left(p_{1}+p_{2}\right)^{2}-p_{3}^{2}\right]^{2} / \lambda^{4}}$. Note that operators $a=a_{\infty}$ are changed to $a_{\lambda}$. In order to see the corresponding non-locality in position variables on the LF hyperplane, we need the notation for quantum fields that is valid for all values of $\lambda[15]$. In this notation,

$$
\int[p]=\int_{-\infty}^{+\infty} \frac{d p^{+}}{4 \pi\left|p^{+}\right|} \iint_{-\infty}^{+\infty} \frac{d^{2} p^{\perp}}{(2 \pi)^{2}}
$$

the effective quantum field is written as $\psi_{\lambda}(x)=\int[p] a_{\lambda p} e^{-i p x}$, and one takes advantage of the identification $a_{\lambda-p}=a_{\lambda p}^{\dagger}$ for positive $p^{+}$. The standard commutation relations take the form $\left[a_{\lambda p}, a_{\lambda_{q}}^{\dagger}\right]=2 p^{+}(2 \pi)^{3} \delta^{3}(p+q)$ and $\left[\psi_{\lambda}(x), \partial^{+} \psi_{\lambda}(y)\right]=i \delta^{3}(x-y)$. Now, using the inverse expression for an annihilation operator for an effective particle with a definite momentum in terms of a quantum field as a function of position variables on the LF,

$$
a_{\lambda p}=\left|p^{+}\right| \int d^{3} x e^{+i p x} \psi_{\lambda}(x)
$$

RGPEP allows one to arrive at the following result. If a canonical Hamiltonian interaction term is of the form

$$
H_{I}=g \int d^{3} x: \psi^{3}(x):
$$

then the renormalized Hamiltonian interaction term of the first order in the effective coupling constant at scale $\lambda$ is of the form given in Eqs. (2.4) and (2.5) with

$$
\hat{f}_{\lambda}\left(x_{1}, x_{2}, x_{3}\right)=\int\left[p_{1} p_{2} p_{3}\right] f_{\lambda}\left|p_{1}^{+} p_{2}^{+} p_{3}^{+}\right| 2(2 \pi)^{3} \delta^{3}\left(p_{1}+p_{2}+p_{3}\right) e^{+i\left(p_{1} x_{1}+p_{2} x_{2}+p_{3} x_{3}\right)} .
$$

In these expression, all momentum variables, including $p^{+}$, are integrated from $-\infty$ to $+\infty$. 
It is clear that if $f_{\lambda}$ in Eq. (2.13) approaches 1 for $\lambda \rightarrow \infty$, this limit renders a local interaction with $\hat{f}_{\infty}\left(x_{1}, x_{2}, x_{3}\right)=\delta^{3}\left(x_{1}-x_{3}\right) \delta^{3}\left(x_{2}-x_{3}\right)$. The reason is that one integrates a plain wave over all values of momenta. However, the form factor $f_{\lambda}$ in Eq. (2.9) was defined in terms of invariant masses of particles with positive momenta $p^{+}$that were limited by momentum conservation, $p_{1}^{+}$ $p_{2}^{+}=p_{3}^{+}$, and thus $p_{1}^{+}$and $p_{2}^{+}$ranged only from 0 to $p_{3}^{+}$. The key question is what function $f_{\lambda}$ appears in Eq. (2.13).

The answer [15] can be stated using a change of variables such that $p_{3}=-P$,

$$
\begin{aligned}
& p_{1}^{+}=\zeta P^{+}, \quad p_{1}^{\perp}=\zeta P^{\perp}+\kappa^{\perp}, \quad p_{1}^{-}=\frac{p_{1}^{\perp 2}+m^{2}}{\zeta P^{+}}, \\
& p_{2}^{+}=(1-\zeta) P^{+}, \quad p_{2}^{\perp}=(1-\zeta) P^{\perp}-\kappa^{\perp}, p_{2}^{-}=\frac{p_{2}^{\perp 2}+m^{2}}{(1-\zeta) P^{+}},
\end{aligned}
$$

and all components of momenta $p_{1}, p_{2}$, and $p_{3}$ range from $-\infty$ to $+\infty$. Then, $f_{\lambda}$ in Eq. (2.13) is given by the same formula as in Eq. (2.9), namely $f_{\lambda}=e^{-\left(\Delta \mathscr{M}^{2} / \lambda^{2}\right)^{2}}$, except that

$$
\left|\Delta \mathscr{M}^{2}\right|=\left[\frac{\kappa^{\perp 2}+m^{2}}{\zeta(1-\zeta)}-m^{2}\right] \frac{1+|\zeta|+|1-\zeta|}{2}
$$

and $-\infty<\zeta<+\infty$. Note that for $\zeta$ in the range between 0 and 1 one has $\zeta=x_{B j}$ and $\frac{1+|\zeta|+|1-\zeta|}{2}=$ 1. The full answer for $\hat{f}_{\lambda}\left(x_{1}, x_{2}, x_{3}\right)$ reads

$$
\hat{f}_{\lambda}\left(x_{1}, x_{2}, x_{3}\right)=\int \frac{d^{3} P}{2(2 \pi)^{3}} \int_{-\infty}^{+\infty} \frac{d \zeta\left|P^{+}\right|}{2(2 \pi)} \int \frac{d^{2} \kappa^{\perp}}{(2 \pi)^{2}} f_{\lambda} e^{-i Y}
$$

where $Y=(\zeta P+\kappa) x_{1}+[(1-\zeta) P-\kappa] x_{2}-P x_{3}$ and $f_{\lambda}=e^{-\left(|\Delta \mathscr{M}|^{2} / \lambda^{2}\right)^{2}}$. The form factor $f_{\lambda}$ tends point-wise to 1 when $\lambda$ tends to $\infty$ and this is how one obtains a local theory from LF Hamiltonians renormalized using RGPEP.

\section{Relationship between non-local vertices and wave functions}

The wave function for a bound state of two constituents at scale $\lambda$ with total momentum $P$, denoted by $\psi_{\lambda P}$, two-body propagator $G_{0}$, and bound-state vertex function $\phi_{\lambda P}$, are combined in the formula $\psi_{\lambda P}=G_{0} \phi_{\lambda P}$. The simplest relationship between a bound-state vertex function and a Hamiltonian interaction term is obtained in the case of factorized interactions. In this case, the bound-state wave function in momentum space is a product of an energy denominator and a factor from the interaction Hamiltonian. Field-theoretic models that exhibit such relation are of the type of the Lee model [17], including its LF counterparts [18]. Using this analogy, the three fields renormalized interaction Hamiltonian term described in Section 2 can be looked at in position space as a vertex function for a bound state of particles 1 and 2 [15]. In what follows, particles 1 and 2 are considered different from each other but have equal masses $m$ and particle 3 is considered to be their bound state of the same mass $m$. This allows us to take advantage of results described in previous sections for a single field $\psi$. When the bound state has momentum $P$, which means definite $P^{+}$and $P^{\perp}$, its vertex function has the form

$$
\phi_{\lambda P}\left(x_{1}, x_{2}\right)=3 g_{\lambda} \int d^{3} x_{3} \bar{f}_{\lambda}\left(x_{1}, x_{2}, x_{3}\right) e^{-i P x_{3}},
$$


where $\bar{f}_{\lambda}\left(x_{1}, x_{2}, x_{3}\right)$ is the part of $\hat{f}_{\lambda}\left(x_{1}, x_{2}, x_{3}\right)$ that results from limiting the integration range in it to positive $P^{+}$and $\zeta$ between 0 to 1 . The factor 3 in front is kept here because it appears in Eqs. (2.8) and (2.9) in the Hamiltonians.

The resulting vertex functions take simple forms in two special cases. In one case, masses of particles 1 and 2 are negligible, which means that $\lambda \gg m \rightarrow 0$ and one considers a situation in which two practically massless partons form a bound state. In this case one obtains

$$
\phi_{\lambda P}\left(x_{1}, x_{2}\right)=3 g_{\lambda}\left(\frac{\lambda}{4 \pi}\right)^{2} P^{+} e^{-i P R} \int_{0}^{1} d z z(1-z) e^{-i(z-1 / 2) \operatorname{Pr}-\frac{1}{4} z(1-z) \lambda^{2} r^{\perp 2}},
$$

where $r=x_{1}-x_{2}$ and both $x_{1}$ and $x_{2}$ are on the same LF with $x^{+}=0 . R=\left(x_{1}+x_{2}\right) / 2$, since we consider here particles 1 and 2 of equal masses. In the other case, the constituents are considered heavy, which means that $\lambda \lesssim m$, and the resulting vertex function corresponds to a constituent model of a meson. In this case, it has the form

$$
\phi_{\lambda P}\left(x_{1}, x_{2}\right)=3 g_{\lambda}\left(\frac{\lambda}{4 \pi}\right)^{2} P^{+} e^{-i P R} C(\lambda / m) e^{-\frac{\lambda^{4}}{96 m^{2}}\left[\left(\frac{P r}{2 m}\right)^{2}+r^{\perp 2}\right]}
$$

where $C(\lambda / m)$ is a number [15]. These vertex functions exhibit dependence on two invariants, $P r$ and $r^{2}$, in a general form of $\phi_{\lambda P} \sim P^{+} \lambda^{2} e^{-i P R} \phi_{\lambda}\left(P r, r^{2}\right)$ valid for $r^{+}=0$. Note that the RGPEP scale parameter $\lambda$ enters the vertex in different ways in ultra-relativistic and non-relativistic cases, since in the latter case the particle mass parameter contributes to the relative motion wave function. The motion of a bound state as a whole is described fully relativistically and in the same way in both cases, by a plane wave.

\section{Conclusion}

One can visualize non-local renormalized interaction Hamiltonian densities for QFTs on the LF using functions of space-time co-ordinates $x^{-}$and $x^{\perp}$ that can be interpreted as positions of constituents of bound states despite that momentum variables $p^{+}=x P^{+}$are not simply related through a Fourier transform to position variables. A wave function of a bound state of momentum $P$ depends on invariants $P r$ and $r^{\perp}$, where $r$ denotes the relative position of the constituents on the LF. Thus, for example, when constructing potentials in LF QCD a la [9], one needs to consider products $P x$ instead of only $p^{+} x^{-}$. The RGPEP scale $\lambda$ enters the relative motion wave functions in different ways for massless and massive constituents. The limit $\lambda \rightarrow \infty$ takes a renormalized non-local interaction to a local one.

\section{Acknowledgement}

The author would like to thank the organizers of LC2010 in Valencia for invitation to speak and outstanding hospitality.

\section{References}

[1] S. Weinberg, Phys. Rev. 150, 1313 (1966).

[2] K. Bardakci, M. B. Halpern, Phys. Rev. 176, 1686 (1968). 
[3] S. D. Drell, D. J. Levy, T.-M. Yan, Phys. Rev. Lett. 22, 744 (1969)

[4] R. P. Feynman, Phys. Rev. Lett. 23, 1415 (1969).

[5] J. D. Bjorken, J. B. Kogut, D. E. Soper, Phys. Rev. D 3, 1382 (1971).

[6] D. E. Soper, Phys. Rev. D 15, 1141 (1977).

[7] S.-J. Chang, R. G. Root, T.-M. Yan, Phys. Rev. D 7, 1133 (1973).

[8] S.-J. Chang, T.-M. Yan, Phys. Rev. D 7, 1147 (1973).

[9] K. G. Wilson et al., Phys. Rev. D 496720 (1994).

[10] G. F. de Téramond, S. J. Brodsky, Phys. Rev. Lett. 102, 081601 (2009) and references therein.

[11] S. S. Gubser, I. R. Klebanov, A. M. Polyakov, Phys. Lett. B 428, 105 (1998).

[12] A. M. Polyakov, Int. J. Mod. Phys. A 14, 645 (1999); Eq. (39).

[13] O. Aharony et al., Phys. Rept. 323, 183 (2000).

[14] S. D. Głazek, Acta Phys. Polon. B 39, 3395 (2008).

[15] S. D. Głazek, Acta Phys. Polon. B 41, 1937 (2010).

[16] F. Wegner, Ann. Phys. (Leipzig) 3, 77 (1994).

[17] T. D. Lee, Phys. Rev. 95, 1329 (1954).

[18] T. Masłowski, M. Więckowski, Phys. Rev. D 57, 4976 (1998). 